\title{
Estratégias e barreiras encontradas pela equipe de enfermagem na comunicação com pacientes deficientes auditivos
}

\section{RESUMO}

Objetivo: identificar e analisar as estratégias e barreiras encontradas pela equipe de enfermagem na comunicação com pacientes deficientes auditivos Método: Revisão integrativa, baseado em artigos científicos publicados até julho de 2019 nas bases de dados PubMed, LILACS, BDENF-Enfermagem e MEDLINE. Os descritores foram selecionados a partir do DeCS, sendo: "Pessoas com Deficiência Auditiva", Comunicação e "Equipe de enfermagem" e no Mesh para o inglês. Não houve restrição de idioma e foram excluídos estudos de revisão de literatura, livros, capítulos e resenhas de livros, manuais e relatórios técnicos. Resultados: As barreiras linguísticas representam o maior dificultante na comunicação entre profissional de enfermagem e o deficiente auditivo. 0 uso de mimetismo e linguagem corporal têm sido utilizados como estratégias. Ofertar disciplinas na formação profissional, bem como disponibilizar treinamentos e protocolos para obtenção de intérpretes podem ser eficazes no processo de comunicação com deficientes auditivos. DESCRITORES: Pessoas com Deficiência Auditiva; Comunicação; Equipe de enfermagem.

\section{ABSTRACT}

Objective: Identify and analyze the strategies and barriers encountered by the nursing staff in communicating with hearing impaired patients. Method: Integrative review, based on scientific articles published until July 2019 in the PubMed, LILACS, BDENF-Nursing and MEDLINE databases. The descriptors were selected from DeCS, namely: "Persons with Hearing Impairments", "Communication", "Nursing, Team" and Mesh for English. There was no language restriction and literature review studies, books, chapters and book reviews, manuals and technical reports were excluded. Results: Language barriers represent the biggest difficulty in communication between nursing professionals and the hearing impaired. The use of mimicry and body language have been used as strategies. Providing subjects in vocational training as well as providing training and protocols for interpreting can be effective in the process of communicating with the hearing impaired.

KEYWORDS: Persons with Hearing Impairments; Communication; Nursing, Team.

\section{RESUMEN}

Objetivo: identificar y analizar las estrategias y barreras encontradas por el personal de enfermería para comunicarse con pacientes con discapacidad auditiva Método: revisión integral, basada en artículos científicos publicados hasta julio de 2019 en las bases de datos PubMed, LILACS, BDENF-Nursing y MEDLINE. Los descriptores se seleccionaron de DeCS, a saber: "Personas con Deficiencia Auditiva", "Comunicación" y "Grupo de Enfermería" y Mesh para inglés. No hubo restricciones de idioma y se excluyeron los estudios de revisión de literatura, libros, capítulos y reseñas de libros, manuales e informes técnicos. Resultados: las barreras del idioma representan la mayor dificultad en la comunicación entre los profesionales de enfermería y las personas con discapacidad auditiva. El uso de la mímica y el lenguaje corporal se han utilizado como estrategias. Brindar materias en capacitación vocacional, así como brindar capacitación y protocolos para la interpretación, puede ser efectivo en el proceso de comunicación con las personas con discapacidad auditiva.

PALABRAS CLAVE: Personas con Deficiencia Auditiva; Comunicación; Grupo de Enfermería.

RECEBIDO EM: 26/11/2019 APROVADO EM: 27/11/2019

\section{Meline Rossetto Kron Rodrigues}

Enfermeira. Professora Doutora do Programa de Pós-Graduação Stricto Sensu em Enfermagem da Universidade UNIVERITAS (UNG) Guarulhos, São Paulo. 
Andréa dos Santos Albuquerque Van-dúnem

Enfermeira. Discente do Mestrado Acadêmico em Enfermagem da Universidade UNIVERITAS (UNG) Guarulhos, São Paulo.

\section{Camilla Romero de Andrade}

Discente de Enfermagem da Universidade UNIVERITAS (UNG) Guarulhos, São Paulo.

\section{Larissa Oliveira Santos}

Discente de Enfermagem da Universidade UNIVERITAS (UNG) Guarulhos, São Paulo.

\section{Kelly Cristina Hilarino de Almeida}

Discente de Enfermagem da Universidade UNIVERITAS (UNG) Guarulhos, São Paulo.

\section{Juliana de Oliveira Santos}

Discente de Enfermagem da Universidade UNIVERITAS (UNG) Guarulhos, São Paulo.

\section{Silvana Andréa Molina Lima}

Enfermeira. Professora Associada ao Departamento de Enfermagem da Faculdade de Medicina de Botucatu (FMB), Unesp, Botucatu, São Paulo.

\section{INTRODUÇÃO}

A deficiência auditiva, conhecida também como surdez, é descrita como a diminuição ou a falta de sensibilidade aos sons do cotidiano e sua origem pode ser de nascença ou por doenças posteriormente adquiridas ao longo da vida ${ }^{(1)}$.

A presença da deficiência auditiva pode ser parcial ou total, onde na primeira existe a capacidade de escuta, mesmo que de forma irregular ou com auxílio de um dispositivo de prótese auditiva; e na segunda, a deficiência auditiva total corresponde aqueles que não têm capacidade funcional de ouvir ${ }^{(1-3)}$.

Dados da Organização Mundial da Saúde (OMS) estimam que, em 2005, havia 278 milhões de pessoas no mundo com deficiência auditiva moderada a profunda. Atualmente, cerca de 466 milhões de pessoas no mundo hoje sofrem com problemas auditivos, sendo 34 milhões crianças. A OMS estima que em 2030 o número de afetados possa alcançar 630 milhões ${ }^{(4,5)}$. A deficiência auditiva e sua privação são interpretadas como um distúrbio neurológico, que afeta de forma direta a aprendizagem e a capacidade de comunicação oral ${ }^{(6)}$. A comunicação é a ação ou efeito de comunicar, de transmitir ou receber ideias, conhecimento e mensagem, sendo vital para manter o convívio em harmonia com a sociedade ${ }^{(7)}$.

A audição é o meio do qual se percebem os sons, visto que é um dos meios mais eficientes de interpretação, deste modo, ser portador de deficiência auditiva não significa ter impedimento intelectual, mas sim portadores de uma deficiência e que necessitam de atenção e cuidados. Deste modo, é de extrema necessidade a qualificação de profissionais em relação ao sistema de Língua Brasileira de Sinais - LIBRAS ${ }^{(6-8)}$.

O sistema de LIBRAS não é composto apenas por gestos e mímicas, mas abarca palavras, sinais e expressões que dão o sentido na comunicação com os deficientes auditivos ${ }^{(8,9)}$.

No Brasil, a LIBRAS é reconhecida legalmente pela Lei Federal n. ${ }^{\circ}$ 10.436/02 e em seu Art. $3^{\circ}$ trata das instituições de saúde, onde define que "as instituições públicas e empresas concessionárias de serviços públicos de assistência à saúde devem garantir atendimento e tratamento adequado aos portadores de deficiência auditiva, de acordo com as normas legais em vigor" ${ }^{(10)}$. O Brasil também conta com o Decreto n. ${ }^{\circ} 5.626$, de 22 de dezembro de 2005 , sendo mais conhecido abertamente por regulamentar a lei anterior e corroborar na garantia do direito à saúde das pessoas surdas ou com deficiência auditiva ${ }^{(11)}$.

O sistema de LIBRAS é entendido como um meio de comunicação importante para a interação social, por parte dos deficientes auditivos, a fim de garantir oportunidades pessoais e profissionais, garantindo, deste modo, a inclusão na sociedade $\mathrm{e}^{(12-14)}$.
A enfermagem tem como objetivo realizar a manutenção, promoção e prevenção da saúde ${ }^{(14)}$. A comunicação desenvolvida com o paciente é primordial para identificar suas necessidades, bem como informar e esclarecer sobre procedimentos, promover o relacionamento paciente e equipe multiprofissional e/ou com familiares, desenvolver educação em saúde, trocar de experiências e mudança de comportamentos, entre outros cenários ${ }^{(15-17)}$.

Deste modo, entende-se que na enfermagem a comunicação é ferramenta essencial para fazer um atendimento integral e de qualidade, sendo necessário que a equipe possa receber e transmitir informações de forma clara e objetiva, para que seja estabelecido vínculo do paciente com o profissional, onde ele possa entender e captar as informações necessárias para prestar um atendimento adequado de acordo com as necessidades de cada indivíduo ${ }^{(18)}$.

Para os pacientes que possuem limitações auditivas, pode haver insegurança referente ao atendimento em saúde, considerando as situações de vulnerabilidade. A equipe de enfermagem possui grande importância nesse contexto, onde é esperado com que a equipe desperte o sentimento de confiança, satisfação e segurança no paciente deficiente auditivo com os profissionais envolvidos no atendimento ${ }^{(17-18)}$.

Estima-se que os profissionais de saúde possuam grande dificuldade relacionada à 
comunicação com pessoas com deficiência auditiva, pois existe falta de conhecimento em LIBRAS, causando transtorno e dificuldade no atendimento prestado ${ }^{(19)}$.

Acredita-se que haja falta de preparo dos profissionais da saúde em atender esta população, especialmente os enfermeiros que comandam as equipes de saúde na atenção primária. A falta de qualificação e a escassez de profissionais capacitados para o atendimento desses deficientes são os preliminares para ocorrência de barreiras que interpõem a comunicação, principalmente em atendimentos hospitalares ${ }^{(20)}$.

Considerando que a forma de comunicar, transmitir ou expressar algo para o paciente pode afetar diretamente o resultado desejado pelo enfermeiro, é essencial desenvolver ferramentas que auxiliem estes profissionais, pois é necessário para o exercício da profissão garantindo a qualida$\mathrm{de}^{(13,17,19,21)}$. Deste modo, o objetivo deste estudo é identificar e analisar as estratégias e barreiras encontradas pela equipe de enfermagem na comunicação com pacientes deficientes auditivos por meio da seguinte questão norteadora: Quais são as estratégias e barreiras encontradas pela equipe de enfermagem na comunicação com pacientes deficientes auditivos?

\section{MÉTODOLOGIA}

Para elaboração deste artigo foi utilizado à metodologia da revisão integrativa da literatura, que busca constatar e sumarizar qual a produção científica disponível acerta da temática em questão, com a finalidade conhecer o que se sabe sobre o assunto e subsidiar novos estudos ${ }^{(22)}$.

Para elaboração da revisão, foram concretizadas seis etapas: a primeira etapa constitui-se na definição das questões principais da pesquisa; na segunda etapa foram definidos os critérios de inclusão e exclusão; na terceira etapa foram selecionadas as bases de dados e realizada a busca dos artigos científicos; na quarta etapa foi realizada a análise dos dados; na quinta etapa foi realizada a discussão dos achados e; finalmente na sexta etapa, a síntese da revisão foi apresentada.
A pergunta norteadora desta revisão foi: Quais são as estratégias e barreiras encontradas pela equipe de enfermagem na comunicação com pacientes deficientes auditivos? Para realizar as buscas nas bases de dados, foram utilizados os Descritores em Ciências da Saúde (DeCS) e seus respectivos sinônimos de "Pessoas com Deficiência Auditiva", "Comunicação" e "Equipe de Enfermagem" em português e em inglês foram utilizados os mesmo descritores selecionados a partir do MesH (Figura 1). O acesso às bases de dados virtuais ocorreu no mês de junho do ano de 2019.

A triagem dos artigos elegíveis foi realizada por dois revisores, buscando garantir rigor metodológico na seleção dos artigos nas bases dados. As bases eletrônicas consultas foram Pubmed, Literatura Latino-Americana e do Caribe em Ciências da Saúde (LILACS), Literatura Internacional em Ciências da Saúde (MEDLINE) e Base de Dados de Enfermagem (BDENF) no sítio da Biblioteca Virtual em Saúde (BVS). Foram utili- zados estudos publicados e indexados nas bases de dados acima referidas. Os critérios de inclusão foram: artigos publicados acerca da temática e publicados até a data da busca. Não houve restrição de idioma.

Os critérios de exclusão foram: revisão integrativa e de literatura, livros, capítulos e resenhas de livros, manuais, relatórios técnicos. Também foram excluídos artigos que não possuíam relação com a questão norteadora do estudo.

A triagem e seleção de artigos foram apresentadas por diagrama de fluxo de estudos e, posteriormente, houve extração dos conceitos abordados em cada artigo e os trabalhos de acordo com seu conteúdo, conforme expressa o diagrama de fluxo de estudos selecionados (Figura 1). Os resultados foram apresentados em forma de quadro e discutidos com os achados da literatura. Referente aos aspectos éticos, por tratar-se de estudo secundário da literatura, não foi necessário a aprovação do Comitê de Ética em Pesquisa (CEP).
Figura 1. Diagrama de fluxo dos estudos selecionados para elaboração de revisão integrativa da literatura para análise das estratégias e barreiras encontradas pela equipe de enfermagem na comunicação com pacientes deficientes auditivos. São Paulo, SP, Brasil, 2019

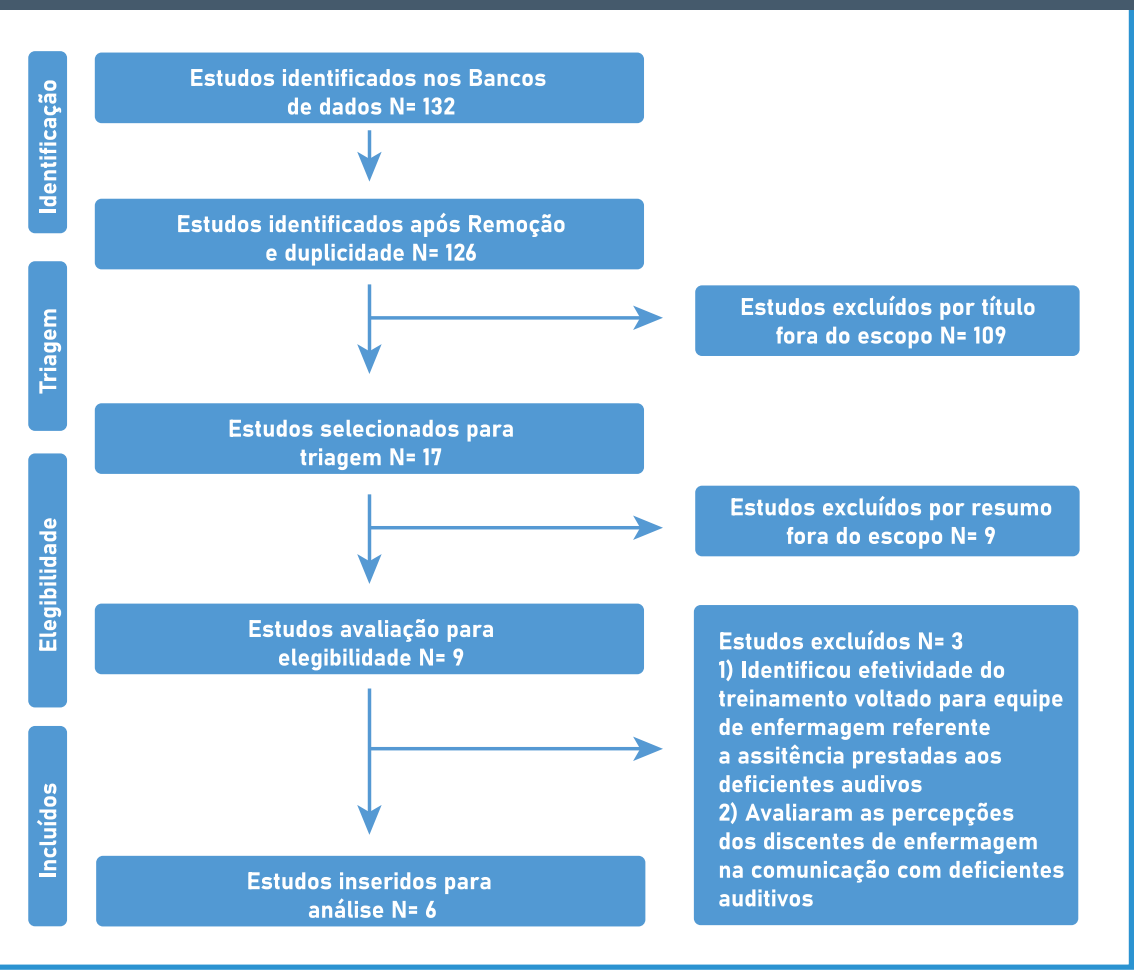




\section{artigo}

Rodrigues, M.R.K.; Van-dúnem, A.S.A.; Andrade, C.R.; Santos, L.O.; Almeida, K.C.H.; Santos, J.O.; Lima, S.A.M.i

Estratégias e barreiras encontradas pela equipe de enfermagem na comunicação com pacientes deficientes auditivos

\section{RESULTADOS}

Nas buscas nas bases de dados, foram resgatados 132 artigos, sendo 125 no Pubmed, 02 na LILACS, 01 no MEDLINE e 04 na BDENF. Inicialmente, foi realizada a triagem por título e 109 artigos foram excluídos nesta etapa. Sequencialmente à triagem, seguiu pela leitura dos resumos e 09 artigos foram excluídos, por fim, 06 artigos foram incluídos para análise. Posteriormente, foram extraídos os conceitos abordados em cada artigo e os trabalhos foram descritos conforme seu conteúdo.

Nesta revisão integrativa da literatura, foram inseridos na análise 06 artigos publicados no período de 2007 a 2018, referentes às estratégias e barreiras encontradas por enfermeiros na comunicação com pacientes deficientes auditivos. Os países de origem das publicações compreenderam Estados Unidos $^{(23,24)}$, Reino Unido ${ }^{(25)}$ e Brasil ${ }^{(26-28)}$. Após análise dos resultados, emergiram duas categorias para análise: 1) Dificuldades e barreiras encontradas por enfermeiros e equipe de enfermagem na comunicação com o deficiente auditivo e 2) Estratégias utilizadas na comunicação de enfermeiros e equipe de enfermagem com o deficiente auditivo:

Os artigos analisados que preencheram os critérios de inclusão previamente estabelecidos, estão sumarizados no Quadro 1, segundo autoria, título do artigo, revista e ano de publicação. O Quadro 2 sumariza os artigos inseridos segundo seus objetivos, instrumento utilizado e síntese dos resultados e conclusões dos estudos analisados.

Quadro 1. Caracterização dos estudos incluídos na análise. São Paulo, SP, Brasil, 2019.

\begin{tabular}{|c|c|c|c|}
\hline & AUTORES & TÍTULO DO ARTIGO & REVISTA/ANO DA PUBLICAÇÃO \\
\hline 01 & Amy L. Ruesch 23 & $\begin{array}{l}\text { Exploring an educational assessment tool to } \\
\text { measure registered nurses' knowledge of hearing } \\
\text { impairment and effective communication strategies: } \\
\text { A USA study }\end{array}$ & $\begin{array}{l}\text { Nurse Education in Practice } 28 \\
\text { (2018) }\end{array}$ \\
\hline 02 & $\begin{array}{c}\text { Kathy Michelle Pendergrass, Lynne S. } \\
\text { Nemeth, Susan D. Newman Carolyn } \\
\text { Jenkins, Elaine G. Jones }{ }^{24}\end{array}$ & $\begin{array}{c}\text { Nurse practitioner perceptions of barriers and } \\
\text { facilitators in providing healthcare for deaf asl users: } \\
\text { a qualitative socio-ecological approach }\end{array}$ & $\begin{array}{l}\text { Journal of the American } \\
\text { Association of Nurse Practitioners } \\
\text { (2017) }\end{array}$ \\
\hline 03 & $\begin{array}{c}\text { Anna Middleton, Alagaratnam Niruban, } \\
\text { Gill Girling, Phyo Kyaw Myint }{ }^{25}\end{array}$ & $\begin{array}{l}\text { Communicating in a healthcare setting with people } \\
\text { who have hearing loss }\end{array}$ & $\begin{array}{l}\text { BMJ: British Medical Journal (Overseas } \\
\text { \& Retired Doctors Edition)(2010) }\end{array}$ \\
\hline 05 & $\begin{array}{c}\text { Lorita Marlena Freitag Pagliucal; Nara } \\
\text { Lígia Gregório Fiúzall; Cristiana Brasil } \\
\text { de Almeida Rebouças }{ }^{27}\end{array}$ & $\begin{array}{c}\text { Aspectos da comunicação da enfermeira com o } \\
\text { deficiente auditivo }\end{array}$ & Rev. esc. enferm. USP (2007) \\
\hline 06 & $\begin{array}{l}\text { Thayana Rose de Araújo Dantas, } \\
\text { Thayris Mariano Gomes,Tatiana } \\
\text { Ferreira da Costa, Thalita Rodrigues de } \\
\text { Azevedo, Silmery da Silva Brito, Kátia } \\
\text { Nêyla de Freitas Macedo Costa }{ }^{28}\end{array}$ & $\begin{array}{c}\text { Comunicação entre a equipe de enfermagem e } \\
\text { pessoas com deficiência auditiva }\end{array}$ & Rev enferm UERJ (2014) \\
\hline
\end{tabular}

Quadro 2. Descrição dos objetivos, instrumento utilizado e síntese dos resultados e conclusões dos estudos incluídos na análise. São Paulo, SP, Brasil, 2019.

\begin{tabular}{|c|c|c|c|}
\hline & OBJETIVO & \begin{tabular}{|l} 
INSTRUMENTO \\
UTILIZADO NA PESQUISA
\end{tabular} & RESULTADOS PRINCIPAIS \\
\hline 01 & $\begin{array}{l}\text { Desenvolver, validar e testar } \\
\text { uma ferramenta de avaliação } \\
\text { para medir o conhecimento dos } \\
\text { enfermeiros sobre deficiência } \\
\text { auditiva, bem como identificar } \\
\text { aparelhos auditivos e outros dis- } \\
\text { positivos auxiliares de audição, } \\
\text { identificar estratégias efetivas } \\
\text { de comunicação para interagir } \\
\text { com o paciente com deficiência } \\
\text { auditiva e leis e políticas relacio- } \\
\text { nadas à interação e atendimento } \\
\text { de pessoas com deficiência } \\
\text { auditiva em enfermeiros }\end{array}$ & $\begin{array}{l}\text { Questionário desenvol- } \\
\text { vido pelo pesquisador } \\
\text { realizado em um hospital } \\
\text { público de grande porte } \\
\text { na Pensilvânia }\end{array}$ & $\begin{array}{l}\text { Foram entrevistados } 339 \text { enfermeiros. Apenas } 28 \text { entrevistados } \\
\text { já participaram de algum curso sobre deficiência auditiva ( } 8 \% \text { ). } \\
\text { Estratégias de comunicação: Todas as estratégias de comuni- } \\
\text { cação são válidas para pessoas surdas }(98 \%) \text {. Ao falar com um } \\
\text { paciente com deficiência auditiva, gritar ou falar diante de uma } \\
\text { fonte de luz direta, é considerado um obstáculo à leitura labial } \\
\text { bem-sucedida ( } 17 \% \text { ). Posicionar-se de frente para a pessoa, } \\
\text { falando claramente em um tom normal, é o meio de comuni- } \\
\text { cação com a pessoa com deficiência auditiva mais importante } \\
(57 \%) \text {. Solicitar as preferências de comunicação da pessoa com } \\
\text { deficiência auditiva é uma estratégia para otimizar melhor a } \\
\text { comunicação ( } 91 \%) \text {. A maioria dos entrevistados conhecem a lei } \\
\text { federal que proíbe a discriminação contra pessoas com deficiên- } \\
\text { cia auditiva e que exige comunicação eficaz com pessoas hospi- } \\
\text { talizadas com deficiência auditiva, sendo a Lei de Reabilitação de } \\
1973 \text {, vinculada a Lei da Deficiência de } 1990 \text { (15\%). }\end{array}$ \\
\hline
\end{tabular}




\begin{tabular}{|c|c|c|c|}
\hline 02 & $\begin{array}{l}\text { Examinar as percepções dos } \\
\text { profissionais de enfermagem } \\
\text { sobre barreiras e facilitadores na } \\
\text { prestação de cuidados de saúde } \\
\text { para surdos. }\end{array}$ & $\begin{array}{l}\text { Entrevistas semiestrutu- } \\
\text { radas, realizadas individu- } \\
\text { almente via telefone. }\end{array}$ & $\begin{array}{l}\text { Barreiras na prestação de cuidados de saúde a usuários surdos: } \\
\text { barreiras linguísticas (não sabem os "sinais" como idioma" ou } \\
\text { "conhece muito pouca linguagem de sinais"). A frequência com } \\
\text { que os profissionais realizam atendimento, pois não se torna } \\
\text { algo rotineiro dos atendimentos. Barreiras organizacionais: } \\
\text { Falta de aviso prévio sobre a ida dos surdos aos atendimentos } \\
\text { e falta do conhecimento do protocolo para se obter um inter- } \\
\text { prete. Necessário haver uma rede de intérpretes acessiveis aos } \\
\text { atendimentos para serem contatados conforme necessário. } \\
\text { Estratégias utilizadas: Leitura labial, gestos e anotações, se } \\
\text { nenhum intérprete estava presente, porém há sentimento de } \\
\text { assistência prestada com deficiência da parte dos profissionais } \\
\text { Facilitadores: A comunicação via intérpretes foi considerada um } \\
\text { facilitador importante por todos os profissionais Considerações } \\
\text { gerais: Todos os profissionais de enfermagem que tiveram } \\
\text { experiência de atendimento com usuários surdos, tiveram pelo } \\
\text { menos um encontro com um sinal intérprete de língua, embora } \\
\text { não fizesse distinção entre intérpretes profissionais e familiares. } \\
\text { Profissionais de enfermagem se sentiram mais confortáveis no } \\
\text { atendimento com surdos quando eles trazem um intérprete, pois } \\
\text { facilita a comunicação. }\end{array}$ \\
\hline 03 & $\begin{array}{l}\text { Fornecer sumário de evidências de } \\
\text { como atender às necessidades de } \\
\text { comunicação de pacientes surdos. }\end{array}$ & $\begin{array}{l}\text { Consenso de especialis- } \\
\text { tas. }\end{array}$ & $\begin{array}{l}\text { Estratégias utilizadas: Chamar a atenção da pessoa antes de falar; } \\
\text { garantir que a pessoa saiba o que está sendo discutido, encarar } \\
\text { a pessoa enquanto fala; manter contato visual; fala claramente e } \\
\text { um pouco mais devagar que o normal; não gritar, pois distorcerá o } \\
\text { padrão da fala, dificultando a compreensão; não cobrir a boca ao } \\
\text { falar; falar pausadamente; reformular em vez de repetir, indicar } \\
\text { quando você mudar para um novo tópico; usa "sinalização" na } \\
\text { conversa, como "Vou perguntar sobre seus sintomas primeiro e } \\
\text { depois faço um exame físico depois"; usar mímica, gesto e lingua- } \\
\text { gem corporal. }\end{array}$ \\
\hline 04 & $\begin{array}{l}\text { Identificar as dificuldades de } \\
\text { comunicação enfrentadas pela } \\
\text { equipe de enfermagem e aprender } \\
\text { as estratégias desenvolvidas para } \\
\text { a comunicação não oral. }\end{array}$ & $\begin{array}{l}\text { Estudo descritivo, } \\
\text { exploratório com } \\
\text { abordagem quantitativa } \\
\text { (questionário). } 0 \text { grupo } \\
\text { foi constituído por } 37 \\
\text { enfermeiros registrados } \\
\text { e } 63 \text { enfermeiros práticos } \\
\text { licenciados de um hospital } \\
\text { particular. }\end{array}$ & $\begin{array}{l}\text { Dificuldades encontradas na comunicação com os pacientes de- } \\
\text { ficientes auditivos: Dificuldades em explicar assunto de interesse } \\
\text { do paciente foram relatadas por } 57 \% \text { dos enfermeiros e } 72 \% \text { dos } \\
\text { técnicos em enfermagem. Entender o paciente a partir da sua } \\
\text { forma de comunicação foi relatado como dificuldade por } 25 \% \text { dos } \\
\text { técnicos de enfermagem e } 43 \% \text { dos enfermeiros. } 3 \% \text { dos técnicos } \\
\text { de enfermagem relataram não ter dificuldade na comunicação, } \\
\text { pois eram portadores de deficiência auditiva parcial e faziam } \\
\text { uso de aparelho auditivo. Estratégias utilizadas: Mimetismo, } \\
\text { seguido pela leitura labial, utilizada por } 94 \% \text { dos entrevistados; } \\
\text { ajuda de uma pessoa acompanhando o paciente foi utilizado por } \\
65 \% \text {, e comunicação escrita por } 42 \% \text { dos respondentes. Apenas } \\
1 \% \text { utilizou a comunicação através da Língua Brasileira de Sinais } \\
\text { (LIBRAS). }\end{array}$ \\
\hline 05 & $\begin{array}{l}\text { Identificar as dificuldades de comu- } \\
\text { nicação da enfermeira com o surdo, } \\
\text { as estratégias de comunicação que o } \\
\text { profissional adotou, como também } \\
\text { perceber o relacionamento com esta } \\
\text { clientela e as sugestões para melho- } \\
\text { rar a comunicação entre enfermeiros } \\
\text { e surdos. }\end{array}$ & $\begin{array}{l}\text { Descritivo exploratório, } \\
\text { com abordagem qualitati- } \\
\text { va, realizado em quatorze } \\
\text { hospitais da cidade de } \\
\text { Fortaleza-CE }\end{array}$ & $\begin{array}{l}\text { Dificuldades de Comunicação: Ausência de preparo na formação } \\
\text { profissional e no ambiente de trabalho Estratégias de Comuni- } \\
\text { cação: mímica, falar pausadamente, leitura labial, usar a escrita, } \\
\text { recorrer a familia Sentimentos no âmbito do relacionamento } \\
\text { profissional: Lidar com os surdos dificulta um pouco o trabalho, } \\
\text { tentar agir naturalmente, aflitivo Sugestões para melhorar a Co- } \\
\text { municação: Deveria haver divulgação de políticas públicas mais } \\
\text { voltadas para essa deficiência, bem como campanhas dentro } \\
\text { dos hospitais, além de treinamentos, cursos de humanização } \\
\text { hospitalar, palestras educativas bem como maior divulgação do } \\
\text { alfabeto dos surdos. }\end{array}$ \\
\hline
\end{tabular}




\begin{tabular}{|l|l|l|l|}
\hline 06 & $\begin{array}{l}\text { Dificuldades encontradas na comunicação da equipe de enfer- } \\
\text { magem com pessoas com deficiência auditiva: Ausência de quali- } \\
\text { ficação para se comunicar, a não comunicação do paciente ser } \\
\text { deficiente auditivo (não haver aviso prévio). Facilidade encontra- } \\
\text { da na comunicação da equipe de enfermagem com pessoas com } \\
\text { com deficiência auditiva. }\end{array}$ & $\begin{array}{l}\text { Pesquisa descritiva, com } \\
\text { abordagem qualitativa, } \\
\text { ancia auditiva: pessoa com deficiência auditiva adapta-se } \\
\text { realizada com } 23 \text { enfer- } \\
\text { meiros e 21 técnicos de } \\
\text { enfermagem. }\end{array}$ & $\begin{array}{l}\text { na comunicação com pessoas com deficiência auditiva: fazer } \\
\text { leitura labial, buscar por interprete da familia, escrita e gestos, } \\
\text { busca pelo serviço social para procurar a família e alguém que } \\
\text { possa auxiliar na comunicação. Sugestões para melhoria da } \\
\text { comunicação: disciplinas na faculdade que abordassem o tema e } \\
\text { ensinamentos em LIBRAS, Capacitações e cursos no âmbito do } \\
\text { trabalho. }\end{array}$ \\
\end{tabular}

\section{DISCUSSÃO}

\section{Dificuldades e barreiras encontradas por enfermeiros e equipe de enfer- magem na comunicação com o defi- ciente auditivo}

A comunicação eficaz entre enfermeiros e pacientes é parte vital para o cuidado de enfermagem seguro e eficaz. Segundo Ruesch $^{(23)}$, as barreiras de comunicação compreendem à deficiência do profissional com o paciente com utilização de linguagem inapropriada, como por exemplo falar diante da luz direta e gritar com o paciente. Estas condutas inapropriadas dificultam a leitura labial.

Os achados indicam que as barreiras linguísticas representam a maior dificuldade de comunicação entre profissional e paciente. Os fatores que influenciam na falta de conhecimento da linguagem, é a frequência com que os profissionais atendem esses pacientes, não se tornando atendimento rotineiro aos profissionais de enfermagem $^{(24)}$. A falta de aviso prévio sobre a ida dos surdos aos atendimentos de saúde e a falta do conhecimento do para obter um intérprete (protocolo para solicitação de um intérprete), também são citados como barreiras do tipo organizacional. Para contê-las, é sugerida a criação de rede de intérpretes para serem contatados quando houver necessidade ${ }^{(27)}$.

Os achados no estudo de Britto e Samperiz ${ }^{(26)}$ evidenciaram que a dificuldade em entender o paciente a partir da sua forma de comunicação foi maior para os enfermeiros, onde $43 \%$ relatou dificuldade,
Deste modo,

é evidente a

importância

do profissional

estar apto para

entender a demanda

dos deficientes

auditivos, para

estabelecer quais

as necessidades do

paciente e prestar

atendimento de

\section{forma humanizada}

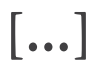

ao passo que apenas $25 \%$ dos técnicos de enfermagem referiu dificuldade na comunicação com este público.

A ausência de preparo na formação profissional e no ambiente de trabalho (ausência de treinamentos e capacitações) foram relatados por dois estudos brasileiros ${ }^{(27,28)}$.

Os pesquisadores ${ }^{(26)}$ encontraram dificuldades na comunicação dos profissionais com os pacientes, relatadas por enfermeiros e técnicos de enfermagem, como a dificuldade em explicar assuntos do interesse do paciente e entender a forma como ele estava se expressando para se comunicar. Apenas $1 \%$ dos profissionais utilizou a língua brasileira de sinais (LIBRAS) para se comunicar com os pacientes surdos.

Deste modo, é evidente a importância do profissional estar apto para entender a demanda dos deficientes auditivos, para estabelecer quais as necessidades do paciente e prestar atendimento de forma humanizada, assim fazendo com que o paciente possa compreender as recomendações necessárias para o tratamento de possíveis doenças e também prover privacidade entre a relação enfermeiro-paciente, visto que muitos intérpretes de LIBRAS são familiares dos mesmos ${ }^{(21)}$.

Estratégias utilizadas na comunicação de enfermeiros e equipe de enfermagem com o deficiente auditivo

A comunicação adequada entre profissional de saúde e usuário é componente fundamental para que se estabeleça interação, acolhimento e vínculo entre eles, colaborando, assim, para que as práticas 
de saúde sejam resolutivas. As pessoas portadoras de deficiência auditiva precisam ser entendidas em suas necessidades e também devem ser alvo das ações de saúde para que, dessa forma, possam vir a exercer conscientemente práticas promotoras, preventivas e curativas de saúde, promovendo sua inclusão social.

Entre as estratégias utilizadas na comunicação de enfermeiros e equipe de enfermagem com o deficiente auditivo, nota-se que a comunicação via intérpretes foi considerada um facilitador importante pelos profissionais da enfermagem ${ }^{(23,26)}$. O estudo de Britto e Samperiz ${ }^{(26)}$ aponta que os profissionais de enfermagem que referiram experiência de atendimento com usuários surdos tiveram, ao menos, um encontro com um intérprete de língua, embora não fizesse distinção entre intérpretes profissionais e familiares. Os profissionais de enfermagem se sentiram mais confortáveis no atendimento com surdos quando eles trazem um intérprete, pois facilita a comunicação.

As estratégias utilizadas pela equipe compreenderam em chamar a atenção da pessoa antes de falar; garantir que a pessoa saiba o que está sendo discutido, encarar a pessoa enquanto fala; manter contato visual; fala claramente e um pouco mais devagar que o normal; não gritar, pois distorcerá o padrão da fala, dificultando a compreensão; não cobrir a boca ao falar e falar pausadamente ${ }^{(24,25)}$. Para Ruesch ${ }^{(23)}$, posicionar-se de frente para a pessoa, falar claramente em um tom normal é o meio de comunicação com a pessoa com deficiência auditiva mais importante.

$\mathrm{O}$ uso de mimetismo e linguagem corporal foi mencionado em cinco $^{(24-28)}$ estudos

\section{A falta de}

conhecimento e

despreparo dos

profissionais para

atender aos pacientes

surdos têm sido

um grande fator

encontrado nos

âmbitos de trabalho

que dificulta a

comunicação

e prejudica a

qualidade e

efetividade no

atendimento.

[... ] como estratégia eficaz utilizada entre deficientes auditivos e equipe de enfermagem.

Como sugestões para melhoria da comunicação, os estudos apontam que devem haver disciplinas na faculdade que abordem o tema e ensinamentos em LIBRAS, bem como capacitações e cursos no âmbito do trabalho ${ }^{(28)}$.

\section{CONCLUSÃO}

A comunicação eficaz entre a equipe de enfermagem e pacientes é essencial para o cuidado de enfermagem seguro e eficaz. As pessoas com deficiência, assim como as demais, têm direito à inclusão nos diferentes ambientes sociais e à uma vida com qualidade com o acesso à educação inclusiva e acessível, além de atenção em saúde de qualidade.

Os achados indicam que as barreiras linguísticas representam grande dificuldade na comunicação entre profissional e paciente. A falta de conhecimento e despreparo dos profissionais para atender aos pacientes surdos têm sido um grande fator encontrado nos âmbitos de trabalho que dificulta a comunicação e prejudica a qualidade e efetividade no atendimento. $\mathrm{O}$ uso de mimetismo e linguagem corporal (linguagem não-verbal) caracterizam-se como estratégias para quebra da dificuldade na comunicação.

Ofertar disciplinas na formação profissional que abordem o tema e auxiliem no preparo profissional da equipe de enfermagem, bem como disponibilizar treinamentos e protocolos para obtenção de intérpretes, podem ser estratégias eficazes para comunicação com deficientes auditivos. -

\section{REFERÊNCIAS}

1. Dantas TRA, GomesII TM, CostallI TF, Azevedo TR, Brito SS, Costa KNFM.Comunicação entre a equipe de enfermagem e pessoas com deficiência auditiva. Rev enferm UERJ. 2014 mar/abr; 22(2):169-74.

2. Marquete VF, Junior LGC, Christinelli H, Teston ÉF, Santos Benedetti GM, Costa MAR. Formação de profissionais para a atenção a saúde de deficientes auditivos. Revista de
Enfermagem da UFSM. 2018; 8(4):649-660.

3. Raimundo RJS, Santos TA. A importância do aprendizado da comunicação em libras no atendimento ao deficiente auditivo em serviço de saúde. Revista Eletrônica de Educação da Faculdade Araguaia. 2012; 3:184-191.

4.Costa MFM. Análise da utilização da Atenção Primária à 


\section{REFERÊNCIAS}

Saúde pelo usuário surdo sob a perspectiva do profissional de enfermagem: estudo descritivo nas unidades de saúde da Ceilândia - DF. 2013. 55 f., il. Monografia (Bacharelado em Saúde Coletiva) - Universidade de Brasília, Ceilândia-DF, 2013

5.Organização Mundial da Saúde [Internet]. União Internacional de Telecomunicações recomendam novo padrão global para prevenir perda auditiva entre 1,1 bilhão de pessoas [acesso em 28 mai 2019]. Disponível em: https://www.paho. org/bra/index.php?option=com_content\&view=article\&id=5869:oms-e-uniao-internacional-de-telecomunicacoes-recomendam-novo-padrao-global-para-prevenir-perda-auditiva-entre-1-1-bilhao-de-pessoas\&Itemid=839.

6. Machado WCA, et al. Integralidade na rede de cuidados da pessoa com deficiência. Texto \& Contexto-Enfermagem. 2018; 27(3).

7.Britto FR, Samperiz MMF. Dificuldades de comunicação e estratégias utilizadas pelos enfermeiros e sua equipe na assistência ao deficiente auditivo. Einstein. 2010; 8(Pt 1):80-5.

8.Pagliuca LMF, Fiúza NLG, Rebouças CBA. Aspectos da comunicação da enfermeira com o deficiente auditivo. Rev. esc. enferm. USP. 2007 Sep; 41(3):411-418.

9. Ferreira GSG, Bicudo EJ, Carvalho DA, Posso MBS, Chagas LR. A Importância da Comunicação no Processo de Enfermagem: A Visão do Enfermeiro. XIII Encontro Latino Americano de Iniciação Científica e IX Encontro Latino Americano de Pós-Graduação - Universidade do Vale do Paraíba, 2009.

10. Brasil. Lei $n .^{\circ} 10.436$, de 24 de abril de 2002. Dispõe sobre a Língua Brasileira .de Sinais - Libras e dá outras providências [Internet]. Brasília (DF), 2002 [acesso em 28 mai 2019]. Disponível em: http://www.planalto.gov.br/ccivil_03/leis/2002//10436.htm.

11. Brasil. Regulamenta a Lei $n .^{\circ} 10.436$, de 24 de abril de 2002, que dispõe sobre a Língua Brasileira de Sinais Libras, e o Art. 18 da Lei no 10.098, de 19 de dezembro de 2000 [Internet]. Brasília (DF), 2000 [acesso em 28 mai 2019]. Disponivel em: http://www.planalto.gov.br/ccivil_03/_ato2004-2006/2005/decreto/d5626.htm.

12. Almeida DL, Lacerda CBF. A escrita de surdos em ambiente internacional de aprendizagem mediado pela LIBRAS. ETD-Educação Temática Digital. 2018; 20(4):10761095.

13.Silva PS, Basso NAS, Fernandes SRCM. A Enfermagem e a utilização da Língua Brasileira de Sinais no atendimento ao deficiente auditivo. Revista UNINGÁ Review. 2014 jan./ mar.; 17(1):05-12.

14. Marquete VF, Costa MAR, Teston EF. Comunicação com deficientes auditivos na ótica de profissionais de saúde. Rev baiana enferm. 2018; 32:e24055.
15. Vasconcelos TAS et al. A comunicação entre ouvintes e pessoas surdas através da LIBRAS nos espaços públicos. Revista Semiárido De Visu. 2017; 4(2):70-76.

16. Nogueira CMI, Carneiro MIN, Da Silva TSA. 0 uso social das tecnologias de comunicação pelo surdo: limites e possibilidades para o desenvolvimento da linguagem. Revista Pesquisa Qualitativa. 2018; 6(12):470-497.

17. Trecossi MO, Ortigara EPF. Importância e eficácia das consultas de enfermagem ao paciente surdo. Revista de Enfermagem. 2013; 9(9):60-69.

18. Oliveira AM, Soares E. Comunicação no relacionamento interpessoal enfermeiro/paciente com indicação de transplante renal. Ciênc. cuid. Saúde. 2016; 15(4):647-654.

19. Francisqueti $V$, et al. Sentimentos da equipe de enfermagem ao atender um deficiente auditivo: desafios do cuidado. Revista Educação, Artes e Inclusão. 2017; 13(3):31-51.

20. Oliveira ECP, Andrade EGS. Comunicação do profissional de enfermagem com o deficiente auditivo. Revista de Divulgação Científica Sena Aires. 2016; 5(1):30-38.

21. Santos NGPS, Silva EGA. Comunicação eficaz através da língua brasileira de sinais do profissional de enfermagem com os deficientes auditivos. Revista de Iniciação Científica e Extensão. 2018; 191):11-17.

22. Mendes KDS, Silveira RCCP, Galvão CM. Revisão integrativa: método de pesquisa para a incorporação de evidências na saúde e na enfermagem. Texto Cont Enferm. 2008; 17(4):758-64.

23. Ruesch AL. Exploring an educational assessment tool to measure registered nurses' knowledge of hearing impairment and effective communication strategies: an USA study. Nurse Education in Practice. 2018 Jun; 28:144-149.

24. Pendergrass KM, Nemeth L, Newman S, Jenkins CM, Jones EG. Nurse practitioner perceptions of barriers and facilitators in providing health care for deaf American Sign Language users: a qualitative socio-ecological approach. J Am Assoc Nurse Pract. 2017; 29(6):316-23.

25. Middleton A, Niruban A, Girling G, Myint PK. Communicating in a healthcare setting with people who have hearing loss. British Medical Journal. 2010; 341:c4672.

26. Britto FR, Samperiz MMF. Dificuldades de comunicação e estratégias utilizadas pelos enfermeiros e sua equipe na assistência ao deficiente auditivo. Einstein (São Paulo). 2010 Mar; 8(1):80-85.

27. Pagliuca LMF, Fiúza NLG, Rebouças CBA. Aspectos da comunicação da enfermeira com o deficiente auditivo. Rev. esc. enferm. USP. 2007 Sep; 41(3):411-418.

28. Dantas TRA, Gomes TM, Costa TF, Azevedo TR, Brito SS, Costa KNFM. Comunicação entre a equipe de enfermagem e pessoas com deficiência auditiva. Rev Enferm UERJ. 2014; 22(2): 169-74. 